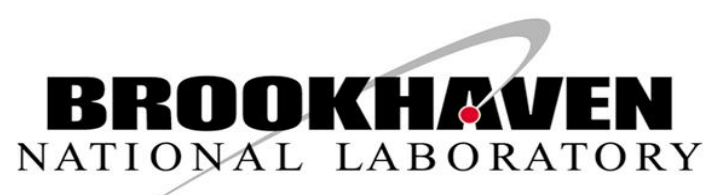

BNL-113988-2017-JA

High-strength magnetically switchable plasmonic nanorods assembled from a binary nanocrystal mixture

Submitted to Nature Nanotechnology

November 2016

Center for Functional Nanomaterials

Brookhaven National Laboratory

\author{
U.S. Department of Energy \\ USDOE Office of Science (SC), \\ Basic Energy Sciences (SC-22)
}

\footnotetext{
Notice: This manuscript has been authored by employees of Brookhaven Science Associates, LLC under Contract No. DE- SC0012704 with the U.S. Department of Energy. The publisher by accepting the manuscript for publication acknowledges that the United States Government retains a non-exclusive, paid-up, irrevocable, world-wide license to publish or reproduce the published form of this manuscript, or allow others to do so, for United States Government purposes.
} 


\section{DISCLAIMER}

This report was prepared as an account of work sponsored by an agency of the United States Government. Neither the United States Government nor any agency thereof, nor any of their employees, nor any of their contractors, subcontractors, or their employees, makes any warranty, express or implied, or assumes any legal liability or responsibility for the accuracy, completeness, or any third party's use or the results of such use of any information, apparatus, product, or process disclosed, or represents that its use would not infringe privately owned rights. Reference herein to any specific commercial product, process, or service by trade name, trademark, manufacturer, or otherwise, does not necessarily constitute or imply its endorsement, recommendation, or favoring by the United States Government or any agency thereof or its contractors or subcontractors. The views and opinions of authors expressed herein do not necessarily state or reflect those of the United States Government or any agency thereof. 


\title{
High-strength magnetically switchable plasmonic nanorods assembled from a binary nanocrystal mixture
}

\author{
Mingliang Zhang, 1,2,3, , Daniel J. Magagnosc², Iñigo Liberal', Yao Yu², Hongseok Yun³, Haoran Yang 2,3,4, \\ Yaoting $\mathbf{W u}^{3}$, Jiacen Guo ${ }^{2}$, Wenxiang Chen', Young Jae Shin ${ }^{1,2,3}$, Aaron Stein', James M. Kikkawa6, \\ Nader Engheta ${ }^{1,2,6,7}$, Daniel S. Gianola ${ }^{2,8}$, Christopher B. Murray ${ }^{2,3 \star}$ and Cherie R. Kagan ${ }^{1,2,3 \star}$
}

\begin{abstract}
Next-generation 'smart' nanoparticle systems should be precisely engineered in size, shape and composition to introduce multiple functionalities, unattainable from a single material ${ }^{1-3}$. Bottom-up chemical methods are prized for the synthesis of crystalline nanoparticles, that is, nanocrystals, with size- and shape-dependent physical properties ${ }^{4-6}$, but they are less successful in achieving multifunctionality ${ }^{7-9}$. Top-down lithographic methods can produce multifunctional nanoparticles with precise size and shape control ${ }^{2,3,10,11}$, yet this becomes increasingly difficult at sizes of $\sim 10 \mathrm{~nm}$. Here, we report the fabrication of multifunctional, smart nanoparticle systems by combining top-down fabrication and bottom-up self-assembly methods. Particularly, we template nanorods from a mixture of superparamagnetic $\mathrm{Zn}_{0.2} \mathrm{Fe}_{2.8} \mathrm{O}_{4}$ and plasmonic $\mathrm{Au}$ nanocrystals. The superparamagnetism of $\mathrm{Zn}_{0.2} \mathrm{Fe}_{2.8} \mathrm{O}_{4}$ prevents these nanorods from spontaneous magnetic-dipole-induced aggregation, while their magnetic anisotropy makes them responsive to an external field. Ligand exchange drives Au nanocrystal fusion and forms a porous network, imparting the nanorods with high mechanical strength and polarization-dependent infrared surface plasmon resonances. The combined superparamagnetic and plasmonic functions enable switching of the infrared transmission of a hybrid nanorod suspension using an external magnetic field.
\end{abstract}

The fabrication of hybrid nanocrystal-based nanorods combines lithographic definition of templates and bottom-up assembly of nanocrystals (Fig. 1a and Methods). These nanorods are assembled from a mixture of $12.6 \pm 0.5 \mathrm{~nm} \mathrm{Zn}_{0.2} \mathrm{Fe}_{2.8} \mathrm{O}_{4}$ nanocrystals ${ }^{12}$ and $4.9 \pm 0.3 \mathrm{~nm} \mathrm{Au}$ nanocrystals ${ }^{13}$ (Supplementary Fig. 1a,b). The long oleylamine ligands capping the Au nanocrystals are completely replaced and the oleylamine/oleic acid ligands capping the $\mathrm{Zn}_{0.2} \mathrm{Fe}_{2.8} \mathrm{O}_{4}$ nanocrystals are $90 \%$ replaced by exchange with compact thiocyanate ligands ${ }^{14}$ (Supplementary Fig. 1c). The fabrication of nanorods on a Durimide layer allows the nanorods to be released and suspended in solvent.

Unless otherwise specified, the $\mathrm{vol} / \mathrm{vol} \mathrm{Zn}_{0.2} \mathrm{Fe}_{2.8} \mathrm{O}_{4}$ :Au nanocrystal ratio in the hybrid nanorods is maintained at $1: 3$. Transmission electron microscopy (TEM) images and elemental mapping reveal complete mixing of the $\mathrm{Zn}_{0.2} \mathrm{Fe}_{2.8} \mathrm{O}_{4}$ and $\mathrm{Au}$ nanocrystals before and after ligand exchange (Supplementary Fig. 2a-c). The sample after ligand exchange shows fusion of the Au nanocrystals to form a porous network ${ }^{15}$. Scanning electron microscopy (SEM) images show that the nanorods in the arrays are $471 \pm 12 \mathrm{~nm}$ in length and $115 \pm 4 \mathrm{~nm}$ in width, as measured from $\sim 60$ nanorods across 3 samples (Fig. 1b,c). The notable size monodispersity is inherited from the control afforded by the templating process. Atomic force microscopy (AFM) measurements show that the nanorods have a 'rabbit ear' morphology (Supplementary Fig. 2d,e) ${ }^{15}$. The nanorod structure remains unchanged after release from the substrate (Fig. 1d). Though assembled from individual nanocrystals, these released nanorods survive a series of harsh solution-based processes (centrifugation and sonication), suggesting strong binding between neighbouring nanocrystals and high nanorod mechanical strength.

To quantify their mechanical properties, we fabricated and mounted 3- $\mu \mathrm{m}$-long hybrid nanorods with the same 1:3 vol/vol $\mathrm{Zn}_{0.2} \mathrm{Fe}_{2.8} \mathrm{O}_{4}$ :Au nanocrystal ratio in a custom in situ nanomechanical testing apparatus (Fig. 2a,b and Methods) ${ }^{16}$. Pure Au nanorods of the same sizes (also nanocrystal-based) were fabricated and characterized for comparison. These nanorods were strained in uniaxial tension until failure (Fig. 2c). Figure 2c (inset) and Supplementary Fig. 3 show representative fracture surfaces observed for both hybrid and pure $\mathrm{Au}$ nanorods, which is uneven and indicative of inelastic deformation.

The stress-strain curves before fracture (Fig. 2d) also show inelastic deformation and strain hardening after the initial linearelastic regime. The average yield/ultimate tensile strengths are $357 \mathrm{MPa} / 706 \mathrm{MPa}$ for hybrid nanorods and $451 \mathrm{MPa} / 741 \mathrm{MPa}$ for pure Au nanorods, respectively. Strikingly, their yield strengths are $>10 \times$ those reported for nanocrystal assemblies (typically $\leq 10 \mathrm{MPa})^{17,18}$, where interdigitation of the nanocrystal surface ligands is postulated to be the dominant strengthening mechanism. Despite the porosity of the nanocrystal-based nanorods (Supplementary Section 1), the measured strength compares favourably with other fully dense Au-based systems such as single-crystal Au nanowires ${ }^{19}$, known for high strengths owing to the scarcity of defects, and freestanding polycrystalline $\mathrm{Au}$ thin films ${ }^{20}$, as well as nanoporous $\mathrm{Au}(\mathrm{np}-\mathrm{Au})^{21}$ (Fig. 2e). The notably high strengths of these nanorods are attributed to their high-density porous Au network (Supplementary Section 2), which ensures the mechanical stability of the nanorods during solution processing.

The magnetic properties of hybrid nanorods were characterized by room-temperature superconducting quantum interference device

\footnotetext{
${ }^{1}$ Department of Electrical and Systems Engineering, University of Pennsylvania, Philadelphia, Pennsylvania 19104, USA. ${ }^{2}$ Department of Materials Science and Engineering, University of Pennsylvania, Philadelphia, Pennsylvania 19104, USA. 'Department of Chemistry, University of Pennsylvania, Philadelphia, Pennsylvania 19104, USA. ${ }^{4}$ The Nature Conservancy, Arlington, Virginia 22203, USA. ${ }^{5}$ Center for Functional Nanomaterials, Brookhaven National Laboratory, Upton, New York 11973, USA. ${ }^{6}$ Department of Physics and Astronomy, University of Pennsylvania, Philadelphia, Pennsylvania 19104, USA. ${ }^{7}$ Department of Bioengineering, University of Pennsylvania, Philadelphia, Pennsylvania 19104, USA. ${ }^{8}$ Materials Department, University of California Santa Barbara, Santa Barbara, California 93106, USA. *e-mail: kagan@seas.upenn.edu; cbmurray@sas.upenn.edu
} 


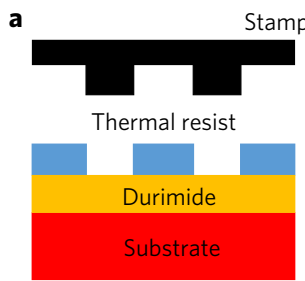

Step 1: nanoimprint

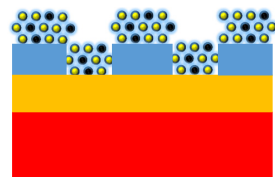

Step 2: nanocrystal deposition

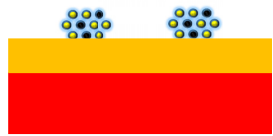

Step 3: lift-off

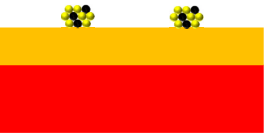

Step 4: ligand exchange

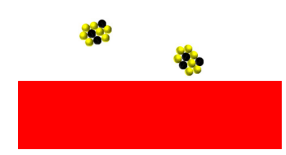

Step 5: release

\section{b

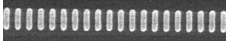

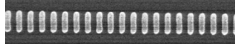

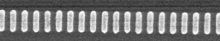

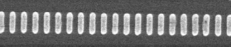

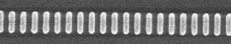
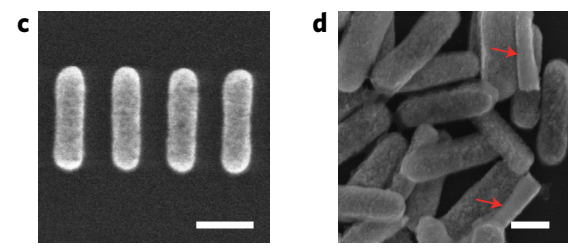

Figure 1 | Fabrication of hybrid nanorods. a, Schematic of the $\mathrm{Zn}_{0.2} \mathrm{Fe}_{2.8} \mathrm{O}_{4}$ :Au hybrid nanorod fabrication process. Step 1: a Si substrate with a spin-coated bilayer of Durimide and thermal resist is imprinted with a nanorod-pillar-patterned stamp. Step 2: a mixture of $\mathrm{Au}$ and $\mathrm{Zn}_{0.2} \mathrm{Fe}_{2.8} \mathrm{O}_{4}$ nanocrystals is deposited by spin-coating. Step 3: resist lift-off. Step 4: ligand-exchange of the patterned nanocrystal-based nanorods with $\mathrm{NH}_{4} \mathrm{SCN}$. Step 5: nanorod release on dissolving the Durimide layer. b,c, Low- and high-resolution SEM images, respectively, of hybrid nanorod arrays before release. d, SEM image of released hybrid nanorods. Red arrows highlight nanorods sitting on the substrate on their sides. Scale bars, $1 \mu \mathrm{m}$ (b); $250 \mathrm{~nm}$ (c); $200 \mathrm{~nm}$ (d).
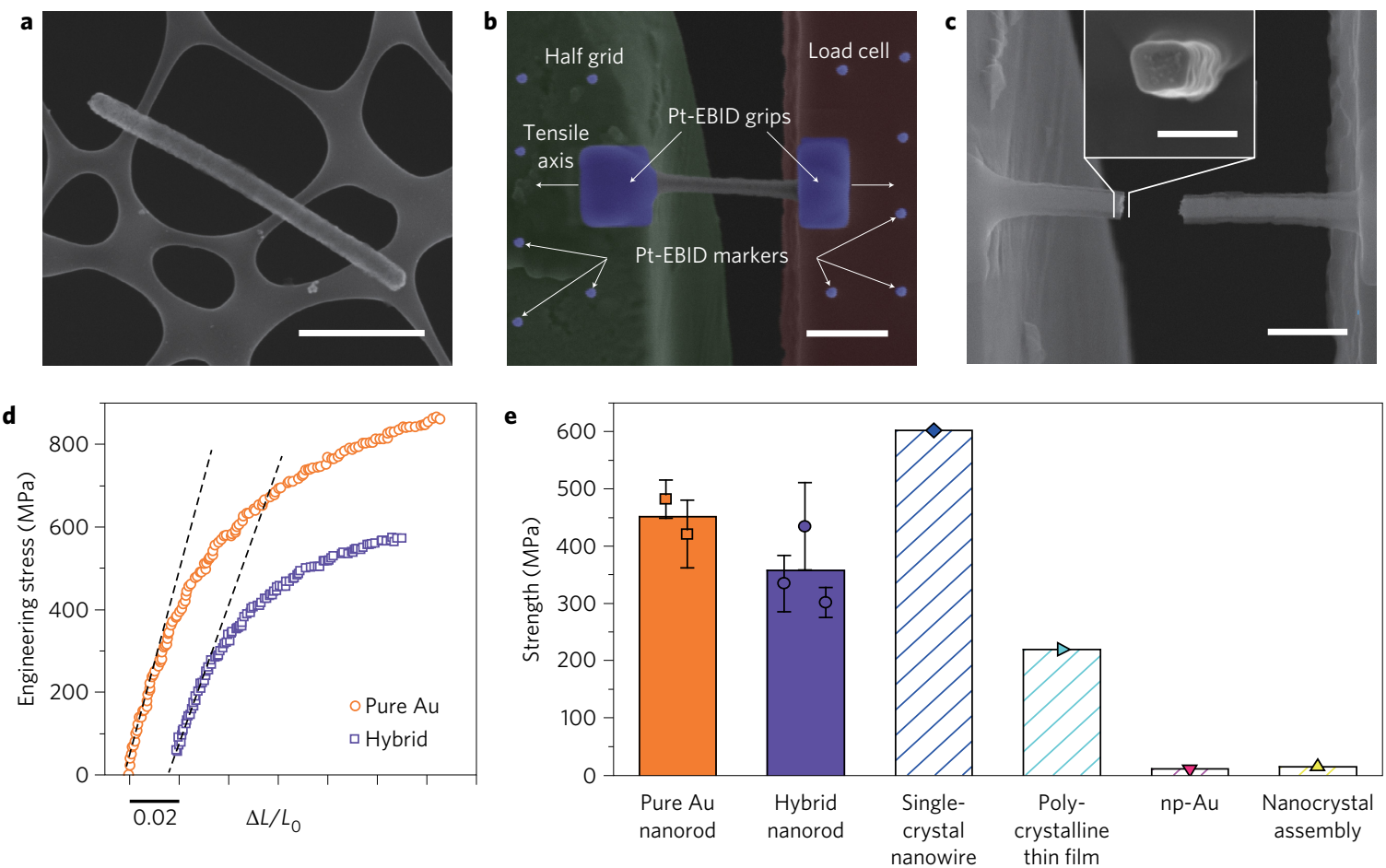

Figure 2 | Mechanical properties of individual nanorods. a, SEM image of a 3- $\mu$ m-long nanorod released and dispersed on a lacey carbon grid for transfer to a nanomechanical testing apparatus. b, SEM image of a manipulated nanorod in which Pt-based electron-beam-induced deposition (Pt-EBID) (blue shading) is used to grip the nanorod between a Cu half grid (green shading) and a microelectromechanical system-based load cell (red shading). c, SEM image of the same nanorod after tensile testing. The inset shows a high-resolution micrograph of a characteristic fracture surface of a nanorod. Scale bars, $1 \mu \mathrm{m}$ (a,b); $500 \mathrm{~nm}$ (c); $250 \mathrm{~nm}$ (c, inset). d, Representative stress-strain curves for pure Au nanocrystal and 1:3 vol/vol $\mathrm{Zn}_{0.2} \mathrm{Fe}_{2.8} \mathrm{O}_{4}: \mathrm{Au}$ hybrid nanorods. Here, $\Delta L$ is the measured change in length and $L_{0}$ is the original gauge length. The curves are offset along the strain axis for clarity. Dashed lines indicate the initial linear elastic response to emphasize the post-yield inelastic behaviour. e, Comparison of strength of the pure Au nanocrystal, hybrid nanocrystal-based nanorods (the error bars represent single measurement uncertainties), single-crystal Au nanowires (diameter $<126 \mathrm{~nm})^{19}$, polycrystalline Au thin films (200 nm thickness) ${ }^{20}$, np-Au (30\% dense, $\sim 30 \mathrm{~nm}$ ligament width) ${ }^{21}$ and monolayer Au nanocrystal assemblies (9.1 nm particle diameter) ${ }^{18}$. Here, the yield/fracture strength value is reported for $\mathrm{np}-\mathrm{Au}$, the fracture strength for the nanocrystal assembly and the yield strength for the single-crystal nanowire and the polycrystalline thin film.

(SQUID) magnetometry (Fig. 3a,b). Arrays of hybrid nanorods with varying $\mathrm{Zn}_{0.2} \mathrm{Fe}_{2.8} \mathrm{O}_{4}$ nanocrystal volume fraction were fabricated on $\mathrm{Si}$ substrates and measurements were conducted in both the longitudinal and transverse direction of the nanorods. Hysteresis loops and blocking temperature measurements (Fig. 3a and Supplementary Fig. 4) showed characteristic superparamagnetic behaviour, in contrast to the noticeable hysteresis in bulk Fe nanorods ${ }^{22}$ and chemically synthesized $\mathrm{Fe}_{3} \mathrm{O}_{4}$ nanorods ${ }^{23}$ of similar size and aspect ratio. While the 

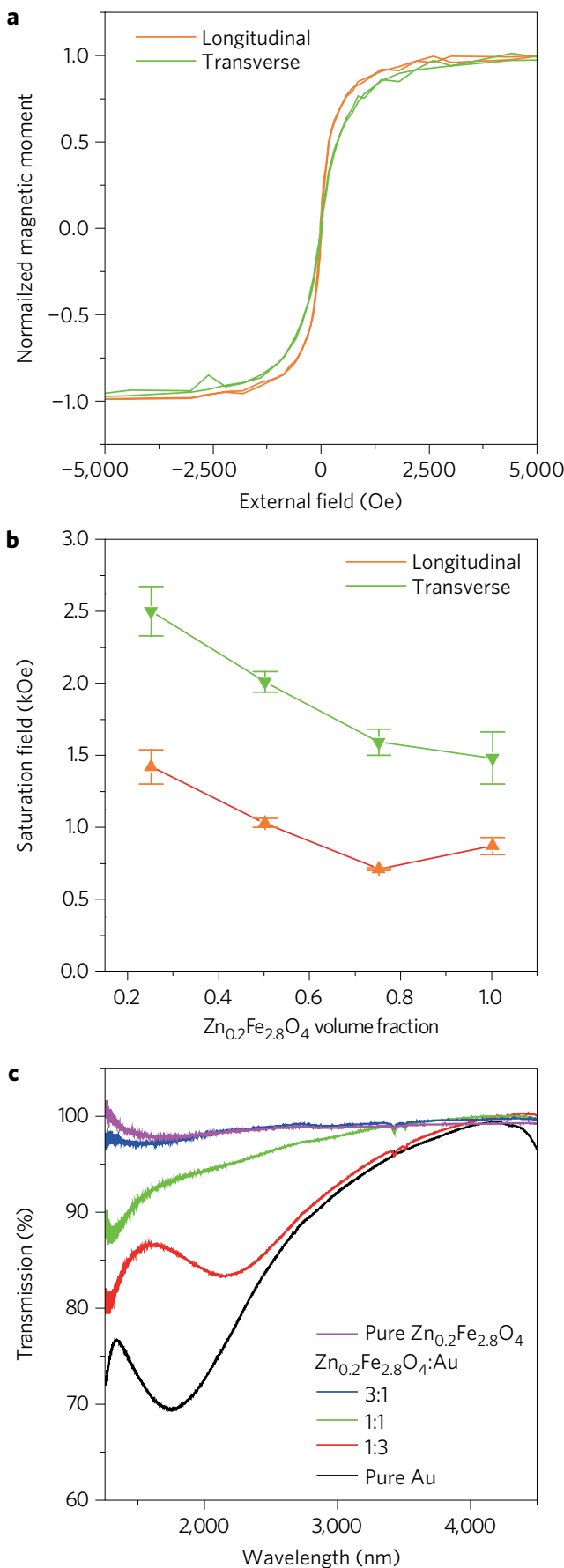

Figure 3 | Magnetic and plasmonic properties of hybrid nanorods. a, Hysteresis loops of a 1:3 vol/vol $\mathrm{Zn}_{0.2} \mathrm{Fe}_{2.8} \mathrm{O}_{4}$ :Au hybrid nanorod array. b. Dependence of the saturation field on the $\mathrm{Zn}_{0.2} \mathrm{Fe}_{2.8} \mathrm{O}_{4}$ nanocrystal volume fraction. The error bars represent single measurement uncertainties. For $\mathbf{a}, \mathbf{b}$, data are collected in the longitudinal and transverse directions. c, Transmission spectra of nanorod arrays with pure $A u$, 1:3, 1:1, 3:1 vol/vol $\mathrm{Zn}_{0.2} \mathrm{Fe}_{2.8} \mathrm{O}_{4}: \mathrm{Au}$ and pure $\mathrm{Zn}_{0.2} \mathrm{Fe}_{2.8} \mathrm{O}_{4}$ nanocrystals illuminated by unpolarized incident light.

overall dimensions of the nanorods are well above the critical dimension for superparamagnetism ( $\sim 25 \mathrm{~nm}$ at $300 \mathrm{~K}$; refs $24-26)$, the superparamagnetic behaviour is consistent with the nanocrystal diameter and the absence of exchange coupling between neighbouring magnetic nanocrystals ${ }^{27}$. Low remanence is advantageous for solutionbased processing, preventing undesired chaining and aggregation ${ }^{28}$ caused by magnetic interactions even in the absence of a magnetic field. Currently, this chaining is usually mitigated by separating the magnetic nanorods with a bulky non-magnetic spacer layer ${ }^{29}$. However, the negligible remanence of these hybrid nanorods makes them naturally immune to chaining, as evidenced by their random orientations (Fig. 1d).

In the presence of an external magnetic field, the magnetic dipolar interactions between nanocrystals lead to magnetic anisotropy associated with the boundary of the nanorods. Demagnetizing factors are smaller for longitudinal magnetic fields than transverse fields, with correspondingly lower saturation fields, regardless of the $\mathrm{Zn}_{0.2} \mathrm{Fe}_{2.8} \mathrm{O}_{4}$ nanocrystal volume fraction (Fig. $3 \mathrm{~b}$ and Supplementary Fig. 5). As a result, the suspended hybrid nanorods can be aligned with the field. The strong magnetic alignment coupled with zero remanence makes the hybrid nanorods promising in applications that exploit control of the nanorod orientation.

The polarization-dependent longitudinal surface plasmon resonance (LSPR) of the nanorods was studied by Fourier-transform infrared spectroscopy. For pure Au nanorods, an electron pathway is established along the Au porous network, leading to metallic $\mathrm{Au}$ optical properties across the entire nanorods ${ }^{14,15}$ (Supplementary Fig. 6a). Consequently, pure Au nanorod arrays fabricated on glass substrates show a LSPR characteristic of their size and shape in the transmission spectrum measured at 1,750 nm (Fig. 3c) and in electromagnetic simulations at $1,600 \mathrm{~nm}$ (Methods and Supplementary Fig. 6b). The LSPR positional difference between experiment and simulation is expected to arise from the rabbit-ear shape of the nanorods.

The incorporation of non-absorbing $\mathrm{Zn}_{0.2} \mathrm{Fe}_{2.8} \mathrm{O}_{4}$ nanocrystals alters the plasmonic properties of the nanorods (Fig. $3 \mathrm{c}$ and Supplementary Fig. 7a). Transmission spectra of similar size and shape hybrid nanorods (Supplementary Fig. $7 \mathrm{~b}, \mathrm{c}$ ) show that with increasing $\mathrm{Zn}_{0.2} \mathrm{Fe}_{2.8} \mathrm{O}_{4}$ nanocrystal volume fraction the position of the LSPR redshifts and its extinction decreases. This trend is consistent with the contribution of the positive dielectric constant of the $\mathrm{Zn}_{0.2} \mathrm{Fe}_{2.8} \mathrm{O}_{4}$ nanocrystals (Supplementary Fig. $7 \mathrm{~d}$ ) in the effective composite medium and a gradual blockage of the electron pathway between $\mathrm{Au}$ nanocrystals by $\mathrm{Zn}_{0.2} \mathrm{Fe}_{2.8} \mathrm{O}_{4}$ nanocrystals. At $>50 \%$ $\mathrm{Zn}_{0.2} \mathrm{Fe}_{2.8} \mathrm{O}_{4}$ nanocrystal volume fraction, the ferrite nanocrystals completely shut down the electron pathway between Au nanocrystals and therefore the LSPR disappears. The percolation threshold provides an upper limit on the $\mathrm{Zn}_{0.2} \mathrm{Fe}_{2.8} \mathrm{O}_{4}$ nanocrystal volume fraction that can be incorporated while still preserving the LSPR.

The selection of 1:3 vol/vol $\mathrm{Zn}_{0.2} \mathrm{Fe}_{2.8} \mathrm{O}_{4}$ :Au hybrid nanorods reflects the balance between requirements of maximizing the magnetic response and of preserving the LSPR resonance. We exploited these nanorods to study the reconfigurable switching of their LSPR by an external magnetic field (the transverse surface plasmon resonance is negligible; Supplementary Fig. 7e). Prior to nanorod release from Durimide, the transmission spectra were collected with linearly polarized incident light, showing a characteristic increase of the LSPR transmission from $51 \%$ to $100 \%$ as the polarization angle $\theta$ was rotated from 0 to $90^{\circ}$ (Fig. 4a,b). The longer-wavelength 2,600-nm LSPR, for nanorods on a Si substrate, in comparison to those on a glass substrate, (Fig. 3c), can be attributed to the difference in substrate dielectric properties and the $\mathrm{Zn}_{0.2} \mathrm{Fe}_{2.8} \mathrm{O}_{4}$ nanocrystal volume fraction variation. After harvesting, these nanorods showed a strong magnetic response. A 750 Oe magnetic field will orient and chain the nanorods in the field direction (Fig. 4c), making them promising building blocks in applications that exploit control of the nanorod orientation.

We released and suspended the hybrid nanorods in Remover PG and loaded the suspension in a liquid cell with $100-\mu \mathrm{m}$ thickness (Supplementary Fig. 8a) and mounted it vertically in the path of linearly polarized light. At this thickness, Remover PG provides a highly transparent medium at wavelengths $<2,700 \mathrm{~nm}$ (Supplementary Fig. $8 \mathrm{~b}$ ). The concentration of the nanorods was estimated to be 


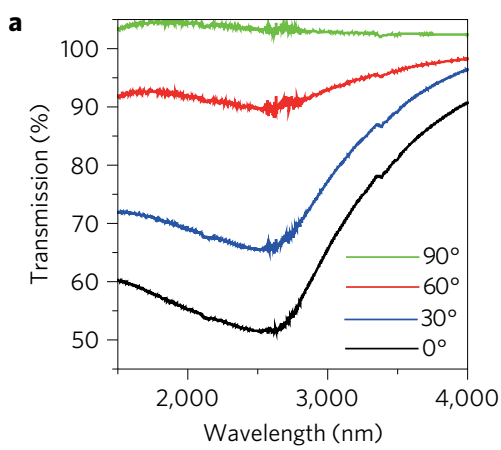

d

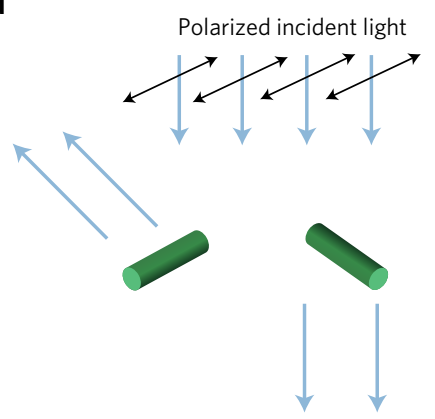

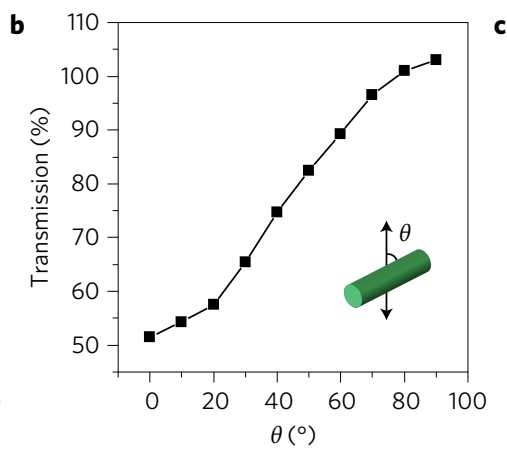
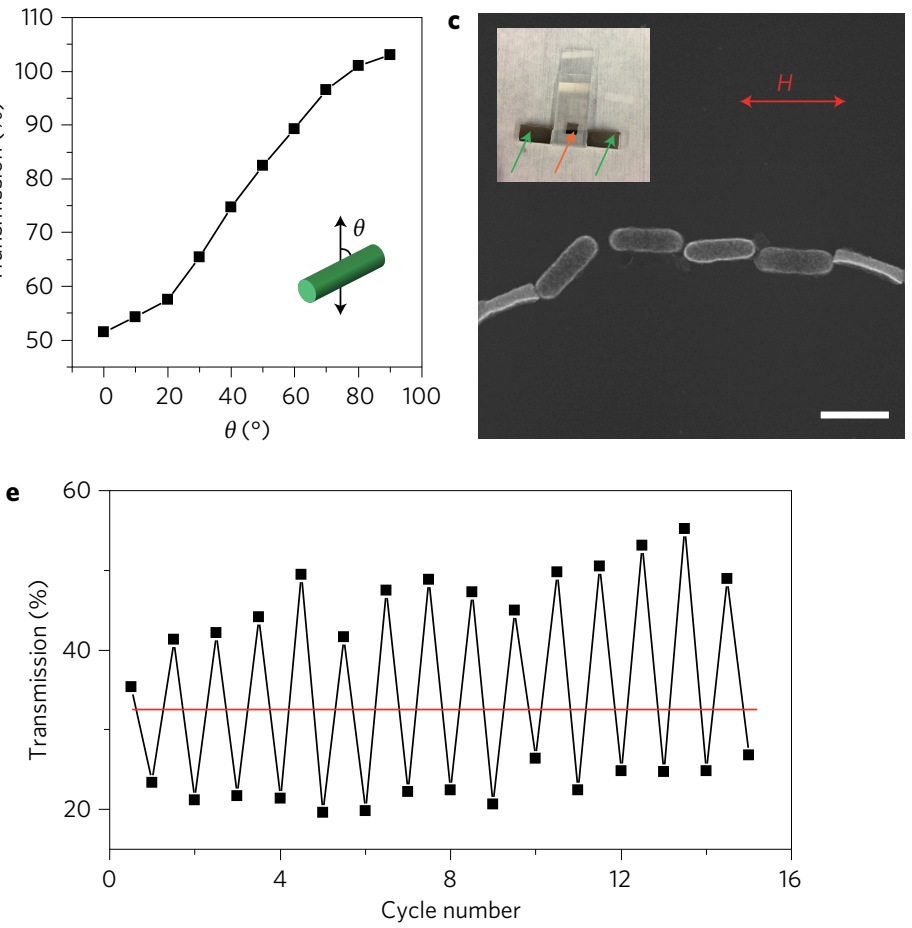

Figure 4 | Modulation of nanorod optical response by an external magnetic field. $\mathbf{a}$, Transmission spectra of the $1: 3$ vol/vol $\mathrm{Zn}_{0.2} \mathrm{Fe}_{2.8} \mathrm{O}_{4}: \mathrm{Au}_{\mathrm{u}}$ hybrid nanorod array as a function of the polarization angle, $\theta$, of incident light from $0^{\circ}$, for light polarized along the longitudinal direction, to $90^{\circ}$, for light polarized along the transverse direction. $\mathbf{b}$, Dependence of nanorod array transmission at $2,600 \mathrm{~nm}$ on $\theta$, the angle between the light polarization direction (black double-headed arrow) and the nanorod (green) orientation. c, SEM image of hybrid nanorods, initially suspended in water and then deposited on a Si substrate under 750 Oe external field, H. Scale bar, $500 \mathrm{~nm}$. Inset shows the sample (orange arrow) in the external field applied by two permanent magnets (green arrows). d, Schematic of the modulated light transmission by controlling the orientation of the hybrid nanorods (green). Black double-headed arrows represent light polarization direction and light blue arrows represent light path. e, Cyclic performance of the light transmission at 2,600 nm for a $100-\mu m$ path length hybrid nanorod suspension in Remover PG under an alternating 120 Oe external magnetic field. Transmission for nanorods oriented perpendicular (above red line) and parallel (below red line) to the light polarization direction.

$3 \times 10^{11}$ nanorods per $\mathrm{ml}$, corresponding to an areal density of 30 nanorods per $\mu \mathrm{m}^{2}$. To avoid magnetic field gradients that drive nanorod migration, a magnet was positioned to the sample side, which resulted in a 120 Oe field. The direction of the external magnetic field was rotated to modulate the orientation of the nanorods, either perpendicular or parallel to the incident light polarization direction, allowing the light to be selectively passed or blocked at the LSPR (Fig. 4d). The rotation was cycled, thereby switching the resonance optical transmission (Fig. 4e and Supplementary Fig. 8c) between average values of $47 \%$ in the passing mode and $23 \%$ in the blocking mode, referenced with respect to a pure Remover PG liquid layer. The $<100 \%$ transmission in the passing mode is consistent with the magnetic hysteresis of the nanorods (Fig. 3a) and suggests the low applied magnetic fields $(120 \mathrm{Oe})$ used are insufficient to saturate the moments and completely align the nanorods. Higher fields can be reached using a more advanced magnetic set-up and promise greater transmission modulation. A trend of slightly increasing transmission in both passing and blocking states as the number of cycles is increased arises from the gradual gravitational sedimentation of the hybrid nanorods, removing nanorods from the light path. However, this process is slow, as only an $\sim 15 \%$ increase of the transmission occurs within the entire experimental observation time of $1 \mathrm{~h}$. More elaborate cell design should be carried out in the future to confine the translational movement of the nanorods while preserving their rotational freedom. Surface functionalization of these nanorods with a highly soluble polymer layer promises to improve their stability in solvent.

In conclusion, by combining top-down and bottom-up fabrication methods we synthesized mechanically robust, hybrid $\mathrm{Zn}_{0.2} \mathrm{Fe}_{2.8} \mathrm{O}_{4}: \mathrm{Au}$ nanocrystal-based nanorods. Ligand-exchange drives fusion of the Au nanocrystals to form a porous network, creating nanorods with high strengths. The synergy of the strong magnetic response introduced by the superparamagnetic $\mathrm{Zn}_{0.2} \mathrm{Fe}_{2.8} \mathrm{O}_{4}$ nanocrystals and the polarization-dependent plasmonic response formed by the porous Au network allows magnetic modulation of the infrared transparency of the hybrid nanorod solution. Compared with previous hybrid systems ${ }^{30-32}$, these hybrid nanocrystal-based nanorods have two advantages: (1) the LSPR may be tuned by lithographic control of the size and shape of the template, providing flexibility in designing the spectral range of transparency modulation; and (2) the sizedependent superparamagnetic character of the ferrite nanocrystals prevents dipole-induced aggregation, in part contributing to the nanorod stability in suspension, and provides for nanorod alignment in an external magnetic field. The nanoimprint process allows the parallel fabrication of large numbers of nanorods and could be scaled up using roll-to-roll processes ${ }^{33}$. These nanorods have potential applications such as smart windows used in buildings to manage indoor light and heat gain and to conserve energy, as well as in targeted photothermal therapies, where the additional magnetic function would enable in vivo nanorod positional control by an external field. More generally, template-based synthesis of nanocrystal-based nanoparticles that combines precise size and shape control and that integrates the physical properties of different nanocrystal building blocks could be useful in the design of multifunctional nanoparticles for multimode bioimaging, targeted drug delivery and magnetically recoverable chemical processing, including water treatment and catalysis. Such a combination is promising in realizing various smart nanoparticle systems. 


\section{Methods}

Methods and any associated references are available in the online version of the paper.

\section{Received 29 March 2016; accepted 29 September 2016;} published online 7 November 2016

\section{References}

1. Kircher, M. F. et al. A brain tumor molecular imaging strategy using a new triplemodality MRI-photoacoustic-Raman nanoparticle. Nat. Med. 18, 829-834 (2012)

2. Perry, J. L., Herlihy, K. P., Napier, M. E. \& Desimone, J. M. PRINT: a novel platform toward shape and size specific nanoparticle theranostics. Acc. Chem. Res. 44, 990-998 (2011).

3. Zhang, M. et al. Magnetically ultraresponsive nanoscavengers for nextgeneration water purification systems. Nat. Commun. 4, 1866 (2013).

4. Murray, C. B., Kagan, C. R. \& Bawendi, M. G. Synthesis and characterization of monodisperse nanocrystals and close-packed nanocrystal assemblies. Annu. Rev. Mater. Sci. 30, 545-610 (2000).

5. Sun, S. et al. Monodisperse $\mathrm{MFe}_{2} \mathrm{O}_{4}(\mathrm{M}=\mathrm{Fe}, \mathrm{Co}, \mathrm{Mn})$ nanoparticles. J. Am. Chem. Soc. 126, 273-279 (2004).

6. Huang, X., Neretina, S. \& El-sayed, M. A. Gold nanorods: from synthesis and properties to biological and biomedical applications. Adv. Mater. 21, 4880-4910 (2009).

7. Ghosh Chaudhuri, R. \& Paria, S. Core/shell nanoparticles: classes, properties, synthesis mechanisms, characterization, and applications. Chem. Rev. 112, 2373-2433 (2012).

8. Wang, C., Xu, C., Zeng, H. \& Sun, S. Recent progress in syntheses and applications of dumbbell-like nanoparticles. Adv. Mater. 21, 3045-3052 (2009).

9. Rivest, J. B. \& Jain, P. K. Cation exchange on the nanoscale: an emerging technique for new material synthesis, device fabrication, and chemical sensing. Chem. Soc. Rev. 42, 89-96 (2013).

10. Sundararajan, S., Lammert, P. E., Zudans, A. W., Crespi, V. H. \& Sen, A. Catalytic motors for transport of colloidal cargo. Nano Lett. 8, 1271-1276 (2008).

11. Zhang, M. et al. High-density 2D homo- and hetero- plasmonic dimers with universal sub-10-nm gaps. ACS Nano 9, 9331-9339 (2015).

12. Yun, H. et al. Alternate current magnetic property characterization of nonstoichiometric zinc ferrite nanocrystals for inductor fabrication via a solution based process. J. Appl. Phys. 119, 113901 (2016).

13. Peng, S. et al. A facile synthesis of monodisperse Au nanoparticles and their catalysis of CO oxidation. Nano Res. 1, 229-234 (2008).

14. Fafarman, A. T. et al. Chemically tailored dielectric-to-metal transition for the design of metamaterials from nanoimprinted colloidal nanocrystals. Nano Lett. 13, 350-357 (2013).

15. Chen, W. et al. Large-area nanoimprinted colloidal Au nanocrystal-based nanoantennas for ultrathin polarizing plasmonic metasurfaces. Nano Lett. 15, 5254-5260 (2015).

16. Magagnosc, D. J. et al. Effect of ion irradiation on tensile ductility, strength and fictive temperature in metallic glass nanowires. Acta Mater. 74, 165-182 (2014).

17. Gauvin, M., Wan, Y., Arfaoui, I. \& Pileni, M. P. Mechanical properties of Au supracrystals tuned by flexible ligand interactions. J. Phys. Chem. C 118, 5005-5012 (2014).

18. Wang, Y. et al. Fracture and failure of nanoparticle monolayers and multilayers. Nano Lett. 14, 826-830 (2014).

19. Sedlmayr, A. et al. Existence of two twinning-mediated plastic deformation modes in Au nanowhiskers. Acta Mater. 60, 3985-3993 (2012).

20. Espinosa, H. D., Prorok, B. C. \& Peng, B. Plasticity size effects in free-standing submicron polycrystalline FCC films subjected to pure tension. J. Mech. Phys. Solids. 52, 667-689 (2004).

21. Balk, T. J., Eberl, C., Sun, Y., Hemker, K. J. \& Gianola, D. S. Tensile and compressive microspecimen testing of bulk nanoporous gold. J. Microsc. 61, 26-31 (2009)

22. Zhang, M., Bechstein, D. J. B., Wilson, R. J. \& Wang, S. X. Wafer-scale synthesis of monodisperse synthetic magnetic multilayer nanorods. Nano Lett. 14, 333-338 (2014).

23. Wang, M., He, L., Zorba, S. \& Yin, Y. Magnetically actuated liquid crystals. Nano Lett. 14, 3966-3971 (2014).
24. Joseyphus, R. J., Narayanasamy, A., Jeyadevan, B., Shinoda, K. \& Tohji, K. Superparamagnetic particle size limit of $\mathrm{Mn}-\mathrm{Zn}$ ferrite nanoparticles synthesised through aqueous method. AIP Conf. Proc. 833, 31-34 (2006).

25. Yang, Y. et al. Synthesis of nonstoichiometric zinc ferrite nanoparticles with extraordinary room temperature magnetism and their diverse applications. J. Mater. Chem. C 1, 2875-2885 (2013).

26. Lv, Z. et al. Magnetic behaviors of $\mathrm{Mg}$ - and $\mathrm{Zn}$-doped $\mathrm{Fe}_{3} \mathrm{O}_{4}$ nanoparticles estimated in terms of crystal domain size, dielectric response, and application of $\mathrm{Fe}_{3} \mathrm{O}_{4} /$ carbon nanotube composites to anodes for lithium ion batteries. J. Phys. Chem. C 119, 26128-26142 (2015).

27. Chen, J. et al. Bistable magnetoresistance switching in exchange-coupled $\mathrm{CoFe}_{2} \mathrm{O}_{4}-\mathrm{Fe}_{3} \mathrm{O}_{4}$ binary nanocrystal superlattices by self-assembly and thermal annealing. ACS Nano 7, 1478-1486 (2013).

28. Zhang, M. et al. Functionalization of high-moment magnetic nanodisks for cell manipulation and separation. Nano Res. 6, 745-751 (2013).

29. Wang, M., He, L., Xu, W., Wang, X. \& Yin, Y. Magnetic assembly and fieldtuning of ellipsoidal-nanoparticle-based colloidal photonic crystals. Angew. Chem. Int. Ed. 54, 7077-7081 (2015).

30. Wang, M. et al. Magnetic tuning of plasmonic excitation of gold nanorods. J. Am. Chem. Soc. 135, 15302-15305 (2013).

31. Mao, Y., Liu, J. \& Ge, J. Tuning the transmittance of colloidal solution by changing the orientation of Ag nanoplates in ferrofluid. Langmuir 28, 13112-13117 (2012).

32. Jung, I., Jang, H. J., Han, S., Acapulco, J. A. I. \& Park, S. Magnetic modulation of surface plasmon resonance by tailoring magnetically responsive metallic block in multisegment nanorods. Chem. Mater. 27, 8433-8441 (2015).

33. Ahn, S. H. \& Guo, L. J. Large-area roll-to-roll and roll-to-plate nanoimprint lithography: a step toward high-throughput application of continuous nanoimprinting. ACS Nano 3, 2304-2310 (2009).

\section{Acknowledgements}

The authors are grateful for primary support of this work from the NatureNet Science Fellowship offered by the Nature Conservancy for nanoparticle fabrication and morphological, optical and magnetic characterization. Electron-beam lithography to pattern the nanoimprint lithography master stamp was carried out at the Center for Functional Nanomaterials, Brookhaven National Laboratory, which is supported by the US Department of Energy, Office of Basic Energy Sciences, under contract no. DE-AC0298CH10886. Optical simulation was supported by the US Air Force Office of Scientific Research MURI grant number FA9550-14-1-0389. Synthesis of Au nanocrystals was supported by National Science Foundation grant no. NSF-561658, and synthesis of $\mathrm{Zn}_{02} \mathrm{Fe}_{28} \mathrm{O}_{4}$ nanocrystals was supported by the Catalysis Center for Energy Innovation, an Energy Frontier Research Center funded by the US Department of Energy, Office of Basic Energy Sciences under award no. DE-SC0001004. Magnetometry was performed in facilities supported by the National Science Foundation MRSEC Program under award no. DMR-1120901. The mechanical testing was supported by the US Department of Energy, Office of Basic Energy Sciences, Division of Materials Science and Engineering under award no. DE-SC0008135. D.J.M. acknowledges the National Science Foundation Graduate Research Fellowship Program under grant no. DGE-1321851 and Y.Y. was supported by University of Pennsylvania's Department of Materials Science and Engineering Masters Scholars Award.

\section{Author contributions}

M.Z., C.R.K. and C.B.M. conceived of and led the project. M.Z. and Y.Y. fabricated the nanorods and performed morphological, optical and magnetic characterization. D.J M. and D.S.G. conducted mechanical characterization. I.L. and N.E. provided optical simulations. Y.Y., H.Yu., H.Ya and Y.W. synthesized the nanocrystals. W.C. and Y.J.S. assisted in the nanofabrication and J.G. contributed to this work in discussions of the nanorod morphology. J.M.K. assisted in the magnetic measurement set-up and A.S. assisted in the electron-beam lithography set-up at Brookhaven National Laboratory.

\section{Additional information}

Supplementary information is available in the online version of the paper. Reprints and permissions information is available online at www.nature.com/reprints. Correspondence and requests for materials should be addressed to C.R.K. and C.B.M.

\section{Competing financial interests}

The authors declare no competing financial interests. 


\section{Methods}

Fabrication and characterization of hybrid $\mathrm{Zn}_{0.2} \mathrm{Fe}_{2.8} \mathrm{O}_{4}$ :Au nanocrystal-based nanorods. The original master stamp for nanoimprint lithography was fabricated by electron-beam lithography over a $1 \mathrm{~cm}^{2}$ area. Briefly, ZEP 520 electron-beam resist was deposited by spin-coating at 3,000 r.p.m. onto a device-grade Si wafer with a $250-\mathrm{nm} \mathrm{SiO} \mathrm{S}_{2}$ layer and baked at $180^{\circ} \mathrm{C}$ for $3 \mathrm{~min}$. The resist layer was patterned with $450 \times 90 \mathrm{~nm}$ nanorods arrayed on an $800 \mathrm{~nm}$ longitudinal and $250 \mathrm{~nm}$ transverse pitch by electron-beam lithography, developed in amyl acetate for $1 \mathrm{~min}$, and rinsed with isopropyl alcohol (IPA). The wafer was then coated with $8-\mathrm{nm} \mathrm{Cr}$ and the resist was lifted-off in Remover PG (MicroChem) at $80^{\circ} \mathrm{C}$ for $2 \mathrm{~h}$. Cr was used as an etch mask to transfer the nanorod array pattern into the $\mathrm{SiO}_{2}$ layer by reactive ion etching (30 mTorr, 38 SCCM Ar, $12 \mathrm{SCCM} \mathrm{CHF}_{3}, 200 \mathrm{~W}$ ) to an etch depth of $180 \mathrm{~nm}$. The as-fabricated master stamp was then treated in an $\mathrm{O}_{2}$ plasma $(70$ mTorr, $70 \mathrm{~W}$, $5 \mathrm{~min}$ ) and soaked in a 1:1,000 $n$-octadecyltrichlorosilane solution in hexane for $10 \mathrm{~min}$ before being used for nanoimprinting. A stamp replication and stitching process was used to enlarge the stamp area, as described in Supplementary Fig. 9.

A Si substrate was spin-coated with Durimide (Durimide 20-500, Fujifilm, 3,000 r.p.m.) and baked at $300{ }^{\circ} \mathrm{C}$, and then spin-coated with thermal resist (thermal imprint resist, NXR-1025, 7\%, Nanonex, 4,500 r.p.m.) and baked at $155^{\circ} \mathrm{C}$. The large-area master stamp was then nanoimprinted into the resist-coated wafer at $133^{\circ} \mathrm{C}$ and 350 psi for 5 min $30 \mathrm{~s}$. Following nanoimprinting, an $\mathrm{O}_{2}$ plasma descum process $(70 \mathrm{mTorr}, 70 \mathrm{~W}, 60 \mathrm{~s}$ ) was used to expose the Durimide layer. A hexane solution containing $\mathrm{Au}$ and $\mathrm{Zn}_{0.2} \mathrm{Fe}_{2.8} \mathrm{O}_{4}$ nanocrystals with different concentrations was deposited by spin-coating onto the substrate at 1,000 r.p.m. for $1 \mathrm{~min}$. The residue nanocrystal layer atop the thermal resist was then lifted-off by immersion in acetone with sonication. The sample was then immersed into a solution of $8 \mathrm{mg} \mathrm{ml}^{-1}$ $\mathrm{NH}_{4} \mathrm{SCN}(99+\%$, Acros) in acetone for $2 \mathrm{~min}$ to exchange the oleylamine surface ligands, followed by rinsing twice in clean acetone. The sample was then dipped in Remover PG with sonication for 5 min to harvest the nanorods. The nanorods were washed in acetone twice and dispersed in distilled water.

Chemicals for nanocrystal synthesis. 1,2,3,4-Tetrahydronaphthalene (98+\%), hydrogen tetrachloroaurate (III) hydrate $\left(\mathrm{HAuCl}_{4} \cdot 3 \mathrm{H}_{2} \mathrm{O}\right)$, zinc (II) acetylacetonate, iron (III) acetylacetonate (99+\%) and 1-octadecene (technical grade, 90\%) were purchased from Acros. Borane-tert-butylamine complex, oleic acid (technical grade, $90 \%$ ) and oleylamine (technical grade, $70 \%$ ) were purchased from Sigma-Aldrich. All the chemicals were used as received.

Au nanocrystal synthesis. Au nanocrystals of $4.9 \mathrm{~nm}$ size were synthesized following literature procedures ${ }^{13}$. Briefly, a reducing solution was prepared by

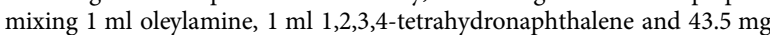
borane-tert-butylamine complex. A precursor solution was prepared by mixing $10 \mathrm{ml}$ oleylamine, $10 \mathrm{ml}$ 1,2,3,4-tetrahydronaphthalene and $100 \mathrm{mg} \mathrm{HAuCl} \cdot 3 \mathrm{H}_{2} \mathrm{O}$ Then the reducing solution was injected into the precursor solution rapidly at room temperature with stirring. The reduction reaction was initiated instantaneously and the colour turned purple within $5 \mathrm{~s}$. The mixed solution was magnetically stirred for $1 \mathrm{~h}$. The Au nanocrystals were collected by adding $60 \mathrm{ml}$ of IPA and followed by centrifugation ( 8,000 r.p.m., $3 \mathrm{~min}$ ) to precipitate the Au nanocrystals. The $\mathrm{Au}$ nanocrystals were re-dispersed in toluene $(7.5 \mathrm{ml})$ and washed by re-precipitating the nanocrystals by adding IPA $(22.5 \mathrm{ml})$ and centrifuging $(8,000$ r.p.m., $3 \mathrm{~min})$. Finally, hexane was used to disperse the Au nanocrystals.

$\mathrm{Zn}_{\mathbf{0 . 2}} \mathrm{Fe}_{2.8} \mathrm{O}_{\mathbf{4}}$ nanocrystal synthesis. $\mathrm{Zn}_{0.2} \mathrm{Fe}_{2.8} \mathrm{O}_{4}$ nanocrystals of $12.6 \mathrm{~nm}$ size were synthesized following literature procedures ${ }^{12}$. Briefly, $2.12 \mathrm{~g}$ of iron (III) acetylacetonate, $790.2 \mathrm{mg}$ of zinc (II) acetylacetonate, $15.9 \mathrm{ml}$ of oleic acid, $19.2 \mathrm{ml}$ of oleylamine and $36 \mathrm{ml}$ of 1 -octadecene were mixed in a $125 \mathrm{ml}$ flask. The reaction mixture was heated to $110^{\circ} \mathrm{C}$ and kept under vacuum. After $2 \mathrm{~h}$, the reaction vessel was filled with nitrogen and the temperature was increased to $300^{\circ} \mathrm{C}$ at a rate of $15^{\circ} \mathrm{C} \mathrm{min}{ }^{-1}$. After $2 \mathrm{~h}$ at $300^{\circ} \mathrm{C}$, the reaction mixture was cooled down to room temperature and the nanocrystals were precipitated by adding a mixture of IPA and ethanol (4:1). The precipitate was readily dispersed in hexane and excess amount of surfactant was removed by further washing with IPA. Finally, the nanocrystals were re-dispersed in $15 \mathrm{ml}$ of hexane and centrifuged to eliminate larger-size nanocrystals.

Hybrid nanorod characterization. The morphology was characterized by SEM (FEI Quanta 600 ESEM for unreleased samples and JOEL 7500F HRSEM for released samples), TEM (JEM-1400 microscope) and AFM (MFP-3D-BIO microscope (Asylum Research Corporation) with an AC240TS silicon cantilever (Olympus)). The optical measurement was conducted by a Nicolet 6700 (Thermo Scientific) Fourier-transform infrared spectrometer with a mercury cadmium telluride detector. For polarization-dependent measurements, linearly polarized excitation was generated by passing the infrared light through a linear infrared polarizer (ThermoFisher Scientific 0045-347). The magnetic characterization was performed using SQUID magnetometory (Quantum Design MPMS) at room temperature, where samples of nanorod arrays on Si substrates were put into straws for measurement.

Characterization of mechanical properties. To characterize the mechanical properties, released nanorods were first drop-cast onto lacey carbon grids. Using pick-and-place nanomanipulation techniques, individual nanorods were detached from the grid and mounted to copper half grids using Pt-based electron-beaminduced deposition (EBID). The free end of the nanorod was attached to the load cell using Pt-EBID and then tested in uniaxial tension under displacement control at a nominal strain rate of $10^{-3} \mathrm{~s}^{-1}$ until failure. Strain was determined using digital image correlation of SEM images obtained in situ by tracking Pt-EBID fiducial markers.

Electromagnetic simulations. Numerical simulations were performed in the $3 \mathrm{D}$ finite-element software COMSOL Multiphysics 5.0, using the frequency-domain solver and a tetrahedral mesh. For the simulation of nanorod arrays, the infinite extent of the arrays is simulated by replicating the basic unit cell with periodic boundary conditions. The unit cell was illuminated by a plane wave under normal incidence and the $\mathrm{SiO}_{2}$ substrate was assumed to be of infinite thickness. The optical constants of the $\mathrm{Au}$ nanocrystals were obtained from ellipsometry measurements (Supplementary Fig. 6). 